\title{
SIRTF, the Space Infrared Telescope Facility
}

\author{
Larry L. Simmons, Jet Propulsion Laboratory, California Institute of Technology
}

\begin{abstract}
The Space Infrared Telescope Facility (SIRTF) is the last of the NASA Great Observatories, and a cornerstone of the NASA Origins Missions. The Observatory will include an $85 \mathrm{~cm}$ telescope in a unique orbit around the sun. The telescope will be launched at ambient temperature and cooled to $5.5 K$ in space. The science instruments will use large detector arrays that will be background limited, and capable of a broad range of astrophysical investigations. The SIRTF architecture will accommodate up to 5 years of crogenic space operations. This talk will describe both the scientific and technical capabilities of SIRTF.
\end{abstract}

\section{Introduction:}

The Space Infrared Telescope Facility (SIRTF) will be the fourth of NASA's Great Observatories following the Hubble Space Telescope (HST), the Compton Gamma Ray Observatory and the recently named Chandra X Ray Observatory. SIRTF is also one of the comerstones of the NASA Origins Program. SIRTF will conclude a two decade effort by the astrophysics community to place into space a facility that will address key questions in astrophysics. How do galaxies form and evolve? What engine drives the most luminous objects in the Universe? Is the mass of the Galaxy hidden in substellar objects? Have planetary systems formed around nearby stars? And possibly most interesting of all, What lies beyond?

\section{The Observatory in Space:}

The SIRTF facility consists of both an Observatory in space and a sophisticated ground system to enable efficient use of the Observatory. The key properties of the Observatory are:

- Telescope: $85 \mathrm{~cm}$ aperture Cassegrain operated at $5.5 \mathrm{~K}$.

- Cryostat: 360 liters of Superfluid Helium at 1.4K.

- Science Instruments:

1. Infrared Array Camera (IRAC), 3.6, 4.5, 5.8, and 8.0 micron bands each with $5 \times 5$ arc minute fields of view.

2. Infrared Spectrograph (IRS), with low resolution spectral capability between $S$ and 40 microns and medium resolution spectral capability between 10 and 40 microns.

3. Multiband Imaging Photometer for SIRTF (MIPS), with 24, 70, and 160 micron imaging capability, in addition to 50 to 100 micron spectral energy distribution capability.

- Pointing: Sub arc second pointing stability and offset accuracy.

- Orbit: Solar, earth trailing.

- Telecommunications: $X$ band up/down using NASA Deep Space Network.

The Observatory, shown below, will be the first large aperture infrared telescope that will be launched at earth ambient temperature, and cooled in space to cryogenic temperature. Cooling will be provided by a combination of radiation to space, and cryogen cooling provided by the vapor from boil off of superfluid helium. This technique will enable a large aperture cryogenic telescope to be put in space with a projected lifetime approaching five years using a Delta II launch vehicle.

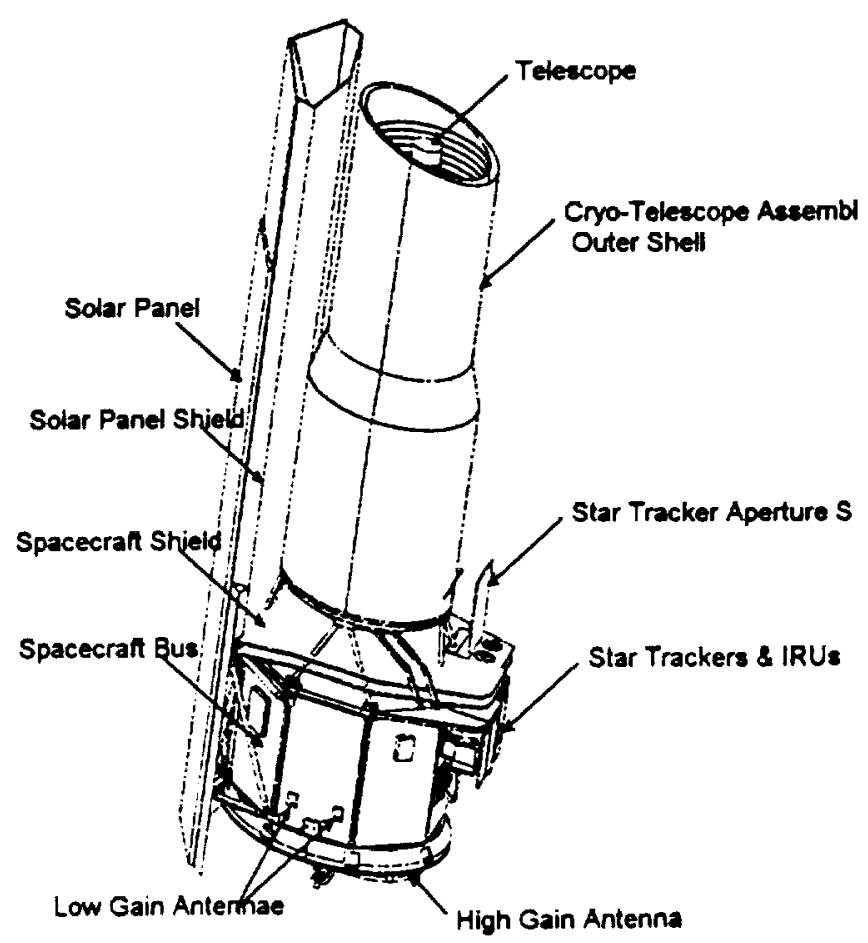


SIRTF will provide a capability to do background limited research in spectral regions not accessible from the earth's surface. Eleven (II) large arrays, sensitive from 3.5 to 180 microns will be available to collect both spatial and spectral information. The unprecedented sensitivity of a new generation of infrared array detectors will enable observations that have been previously unachievable either in space or from the ground. For example, SIRTF will measure sources which are 10,000 times fainter than the limits reached by the IRAS all sky infrared survey in 1983. For simplicity and reliability, SIRTF has only four (4) mechanisms that operate at cryogenic temperatures (not counting a deployable dust cover for the telescope and cryostat valves).

SIRTF will be placed in a solar orbit that will allow the .Observatory to trail the Earth as it orbits the sun. The observatory will recede from the earth at about 0.13 AU/year. At this rate the Observatory is expected to be at about $0.64 \mathrm{AU}$ at the end of the 5 year planned mission. This orbit has many advantages. Among them are; maximum payload to orbit, no need for on-boand propulsion system, no periodic solar eclipse by earth, no thermal radiation from earth, and excellent access to astronomical targets.

The Observatory will communicate with Earth periodically using the NASA Deep Space Network (DSN). Data are stored on board and downlinked every 12 hours. Operation of the Observatory will be conducted by on-board systems responding to preplanned sequences which are sent to the Observatory once a week. Data transmission will use $\mathrm{X}$ band systems, and will have a bit rate for the first 2.5 years of $2.2 \mathrm{Mbps}$. The spacecraft portion of the Observatory also provides solar collected power, pointing control, and command and data handling of the Observatory and its instruments.

The facility will be accessible by the entire international community through the SIRTF Science Center which will solicit investigations, coordinate all scientific observations, and will calibrate and distribute the resulting data. At least $75 \%$ of observing time will be awarded through peer reviewed proposals. Day by day control of the Observatory will be performed by a flight operations system that includes the NASA Deep Space Network, and multimission capabilities at JPL.

\section{The Cryogenic Telescope Assembly:}

Prior to SIRTF, infrared astronomy missions have been designed to cool both the instruments and telescope prior to launch, and then open a cover in space to commence observing. The penalty associated with this approach is that to maintain all of the needed equipment at cryogenic temperatures on the ground requires that they be placed in a vacuum container. As the size of the telescope aperture grows, so does the size (and mass) of the vacuum containcr. For SIRTF, an approach has been developed that allows the $85 \mathrm{~cm}$ Beryllium telescope to be at room temperature on the ground, and then cooled in space to $5.5 \mathrm{~K}$ by a combination of radiation, and superfluid helium boil off. As a result the vacuum container need only contain the cold portions of the instruments and the cryogen.

Radiation cooling is accomplished by shading the Observatory from the sun using the solar array. The warm spacecraft portion of the Observatory is isolated from the cold portion, known as the Cryogenic Telescope Assembly (CTA), using struts made of gamma-alumina. As a result of shielding and isolation, the telescope will cool to less than $40 \mathrm{~K}$ using radiation alone. Added cooling of the telescope is provided by thermally connecting the telescope to the lines carrying the vapor from the boil off of superfluid Helium. A resulting telescope temperature of $5.5 \mathrm{~K}$ is obtained if the heat load on the helium is about 6 milliwatts. Since that is the design point for the cryogenic portions of each instrument, an efficient use of cryogen results. While the requirement for SIRTF is to sustain the cryogenic operations for 2.5 years, current estimates are that under nominal conditions a lifetime of 5 years can be expected with only 360 liters of Helium at launch.

The cryogenic portions of the instruments are contained within a Multiple Instrument Chamber (MIC) located in the cryostat. The cryostat is surrounded by shields designed to minimize the heat load on the cryogen. The shields are vapor cooled and/or radiate to space resulting in a cryostat with an outer vacuum shell operating at near $5 \mathrm{~K}$.

\section{The InfraRed Array Camera (IRAC):}

IRAC is a camera intended to provide high resolution and wide field imagery in four spectral bands. The camera is designed to have two optical paths, and each path contains a beamsplitter that sends short wavelength radiation to a $256 \times 256$ element Indium Antimonide (InSb) array, and the long wavelength radiation to a 256×256 element Arsenic doped Silicon (Si:As) array. One optical path provides images at 3.6 microns and 5.8 microns. The other path provides images at 4.5 microns and 8.0 mictons. The only moving element of the camera is a shutter which will be used occasionally to close the aperture for calibration purposes. The IRAC uses programmed gate arrays to establish operating sequences. Commands from the spacecraft are used to establish bias voltages, and command readouts. The simplicity of the design is considered to be a strength for reliability and case of operation.

The InfraRed Spectrograph (IRS): 
The IRS consists of four modules, each of which is a self contained spectrograph with a $128 \times 128$ element array in the focal plane. Two modules are low resolution spectrographs, one operating between 5 and 15 microns, and the other between 15 and $\mathbf{4 0}$ microns. The other two modules are medium resolution spectrographs operating between 10 and 20 microns, and between 20 and 40 mictons with orders folded to fully utilize the second dimension of each array. Both short wavelength spectrographs are read out using $128 \times 128$ element Si:As arrays. Similarly, at the longer wavelengths $128 \times 128$ element Antimony doped Silicon (Si:Sb) arrays are used.

In order to assure placement of the desired object on a selected spectrograph slit, a portion of one array will be used to form an image to peak-up the object. Peak-up sefers to the process of optimally placing the object on the selected slit. For SIRTF this will be done on-board using information about the location of the object derived from the peak-up array. This information will be processed by the spectrograph. The resulting data will be used to define a pointing offset, and the telescope will then be pointed such that the object falls on the selected spectrograph slit.

\section{The Multiband Imaging Photometer for SIRTF (MIPS):}

MIPS contains three arrays which are used primarily for imaging. It may also be used in a spectral energy distribution mode, and in a photometer mode to measure total power being observed. Unlike the other SIRTF instruments, MIPS is dependent upon a cryogenically cooled scanning mirror for proper operation. The shortest wavelength for MIPS is 24 microns, which is observed using a $128 \times 128$ element $\mathrm{Si}$ :As array. At 70 microns a $32 \times 32$ element Gallium doped Germanium (Ge:Ga) array is employed. For the longest wavelengths being observed by SIRTF, out to about 180 microns, a $2 \times 20$ element Stressed $\mathrm{Ge}: \mathrm{Ga}$ array is used. The MIPS pixels have been sized to permit critical sampling of the image from the 85 $\mathrm{cm}$ telescope. The scanning mirror has several facets, and allows for simultaneous observing with each of the three arrays. By repositioning the scanning mirror additional optics are introduced into the path that disperse the radiation through a low resolution grating resulting in a measurement of spectral energy distribution, the SED mode. Another position of the scanning mirror places MIPS in the total power mode.

The IRS and MIPS instruments use a common set of warm electronics which contains a RAD 6000 microprocessor that controls the operating instrument, and sends the resulting data to the spacecraft for packing, storage, and subsequent transmission to the earth.

\section{The Spacecraft:}

The primary functions of the spacecraft are to provide power, pointing, command and data handling, and telecommunications. Each function requires sophisticated on-board systems to assure high reliability and precision operation. Power is provided by Gallium Arsenide solar cells collecting solar energy and distributing it on a bus to internal subsystems, and on command to the designated instrument. A battery is included, primarily for launch operations, but also for additional protection in the event of an Observatory anomaly.

Pointing is provided using a combination of star tracker data, gyroscope data, and information from a Pointing Calibration Reference Sensor (PCRS) located in the cryogenic focal plane of the telescope. Reaction wheels are used to position the Observatory, with a nitrogen gas reservoir on board for momentum management. Both the star tracker and PCRS are sophisticated instruments which internally determine their pointing direction; and relay this information to the spacecraft computer which uses the data to determine how to point the telescope in the desired direction.

The spacecraft uses a RAD 6000 microprocessor based Command and Data Handling (C\&DH) subsystem that provides the computing power for the entire spacecraft. Instrument commands are issued by the $C \& D H$, and the resulting data is collected, compressed, packetized, and stored for transmission to earth in subsequent telecommunications sessions. Observing sequences are controlled by the C\&DH based on weekly updates from earth. The C\&DH also provides the processing capability needed to point the observatory, and maintain its position during observations and when communicating with earth.

Telecommunications are at $\mathrm{X}$ band frequencies using a 1.35 meter high gain antenna mounted at the base of the spacecraft. The antenna is fixed, and is pointed to the Earth by pointing the entire Observatory. When in communication with the Earth, data transmission occurs at rates up to $2.2 \mathrm{Mbps}$, and commands can be concurrently sent to the Observatory at a rate of $2 \mathrm{Kbps}$. A NASA developed Small Deep Space Transponder (SDST) prepares the packetized on board data for transmission, and sends the Reed-Solomon encoded convolutionally coded data to the X-band solid state power amplifiers. The SIRTF design provides for redundant transmitters at 15 watts that can be connected together for a non-redundant transmission capability of 29 watts. This feature will be used to extend the high data rate capabilities of SIRTF as the mission progresses and the distance from the earth to the Observatory increases.

Key SIRTF Tests prior to launch: 
The reliance on the SIRTF thermal design to achieve the required performance necessitates a robust test program. While the concept of radiative cooling is straight forward, its implementation is sophisticated. To assure that acceptable operating temperatures will be attained, and that the lifetime expected will be achieved, a thermal balance test is planned to demonstrate that the thermal design performs as specified. This test will require the use of a large Helium shroud to simulate the space environment for the entire CTA.

Optical performance is being provided using a technique not previously proven in space. Launching the SIRTF telescope at earth ambient temperature, and then cooling it in space to $5.5 \mathrm{~K}$ while requiring diffraction limited performance (at 6.5 microns) represents a significant optical challenge. To verify that the telescope will meet its requirements, an end-to-end optical test is planned following assembly of the CTA. The telescope will be cooled from earth ambient temperature to $5.5 \mathrm{~K}$ in a test chamber, and IRAC and IRS arrays will be illuminated to demonstrate that the telescope performs as required at cryogenic temperatures. Risk has been reduced by including a focus mechanism at the secondary mirror which will be used for ground testing, and can be used in space if needed.

In addition to these performance tests, a series of operational scenario tests will be conducted on the entire observatory prior to launch to assure that the ground and Observatory systems perform together as expected. The scenarios will verify command and data handling capabilities, the ability of ground systems to plan and load long sequences up to the Observatory, and the ability of the Observatory to execute them autonomously.

\section{The Ground System}

The SIRTF Ground System consists of two main elements; a Flight Operations System (FOS) which has real time responsibility for operation of the Observatory, and the Science Operations System (SOS) with responsibility for making the facility accessible to the user community.

The SOS issues periodic announcements to the user community, selects observations to be performed on the Observatory, and plans observing sequences that efficiently use the Observatory's resources. Once observations are made, and data are returned to earth, the SOS processes the data to remove instrumentation artifacts. The resulting data are delivered to the requesting user(s) and placed in a SIRTF archive for general use by the research community.

The FOS, working with the SOS. prepares weekly sequences for execution on the Observatory. Once agreed upon, the weekly sequences are up loaded to the Observatory concurrent with one of fourteen weekly downlink passes. Two times each day, data are acquired from the Observatory using the NASA DSN. The received data are decoded, unpacked, and the engineering data sent to monitoring teams for evaluation. The science data are sent to the SOS for processing and distribution.

\section{The SIRTF Team:}

SIRTF is being developed using a team approach The Jet Propulsion Laboratory has overall project responsibility and is the team leader, in addition JPL is responsible for the Flight Operation System development. The Smithsonian Astrophysical Observatory is providing the IRAC instrument with hardware being provided by the NASA Goddard Space Flight Center. Comell University is providing the IRS instrument with hardware being provided by the Ball Aerospace and Technology Corporation. The University of Arizona is providing the MIPS instrument with hardware being provided by the Ball Aerospace and Technology Corporation. The Ball Aerospace and Technology Corporation is also providing the Cryogenic Telescope Assembly. Lockheed Martin Missiles and Space is providing the Spacecraft, system engineering support, and Observatory level integration, test and launch coordination. The Science Operation System development is the responsibility of the California Institute of Technology.

Additional information on SIRTF can be obtained by accessing SIRTF information on the World Wide Web at http://SIRTF.caltech.edu.

The research described in this paper was carried out by the Jet Propulsion Laboratory, California Institute of Technology, under a contract with the National Aeronautics and Space Administration. 


\section{AUTHORIZATION FOR THE EXTERNAL RELEASE OF INFORMATION}

Submlt URL (if applicable) or two coples of the abstract or full paper to Document Review, 111.120

CL No. Nor DAs un ony?

\begin{tabular}{|l|l|l|l|l|}
\hline Senior JPL Author & Section & Mall Stop & Ext. \\
& Larry L. Simmons & 7500 & $264-767$ & $4-6336$ \\
\hline
\end{tabular}

Due Date

COMPLETE TITLE

SIRTF, The Space Infrared Telescope Facility

$\square$ Foreign $\square$ Domestic Account Code

ABSTRACT (including extended abstract)

$\bigotimes$ FULL PAPER (including viewgraphs, poster, videocassette)

$\square$ Journal Name

$\triangle$ Meeting - Subject 16th IEEE Instrumentation \& Measurement Technology Conf. Sponsoring Society IEEE

- Meeting Date $05 / 24 / 1999$

Location Venice, Italy

\section{BOOK OR BOOK CHAPTER}

\section{$\square$ Assigned Laboratory Task}

OR

PUBLICATION

$\square$ BROCHURE

URL:

Private Venture $\square$ Premeeting publication

$\square$ Publication on meeting day

$\square$ Postmeoting publication

$\square$ Poster session

$\square$ Oral presentation

Was previously cleared: Clearance No(s).:
CL-
Date
Author(s)
CL-
Date
Author(s)

\section{REPORTABLE INFORMATION}

THIS WORK: $\square$ New technology not previously reported

Nature of this work (please describe)

$\bigotimes$ Covers work previously reported in New Technology Report (NTR) No. various

$\square$ Provides more information for earlier NTR No(s).

FOR TECHNOLOGY REPORTING AND COMMUNICATIONS USE ONLY

Release $\square$ Ex Post Facto

Release Delayed or Conditional

Comments:

\section{FOR SECTION 644 USE ONLY}

Editor

Customer Code (RTOP No.)
Ext. Document No.

Group Condition
AUTHORIZATION (please use blue ink)

The signatory in this column attests to the technical accuracy of the subjoct docurhent.

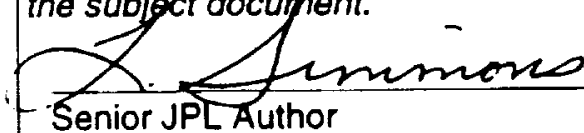

Senior JPL Author $1 / 26 / 99$ Date
Technology Reporting and Communications Date 


\title{
SIRTF, the Space Infrared Telescope Facility
}

\author{
Larry L. Simmons, Jet Propulsion Laboratory, California Institute of Technology
}

\begin{abstract}
The Space Infrared Telescope Facility (SIRTF) is the last of the NASA Great Observatories, and a comerstone of the NASA Origins Missions. The Observatory will include an $85 \mathrm{~cm}$ telescope in a unique orbit around the sun. The telescope will be launched at ambient temperature and cooled to $5.5 \mathrm{~K}$ in space. The science instruments will use lange detector arrays that will be background limited, and capable of a broad range of astrophysical investigations. The SIRTF anchitecture will accommodate up to 5 years of crogenic space operations. This talk will describe both the scientific and technical capabilities of SIRTF.
\end{abstract}

\section{Introduction:}

The Space Infrared Telescope Facility (SIRTF) will be the fourth of NASA's Great Observatories following the Hubble Space Telescope (HST), the Compton Gamma Ray Observatory and the recently named Chandra X Ray Observatory. SIRTF is also one of the comerstones of the NASA Origins Program. SIRTF will conclude a two decade effort by the astrophysics community to place into space a facility that will address key questions in astrophysics. How do galaxies form and evolve? What engine drives the most luminous objects in the Universe? Is the mass of the Galaxy hidden in substellar objects? Have planetary systems formed around nearby stars? And possibly most interesting of all, What lies beyond?

\section{The Observatory in Space:}

The SIRTF facility consists of both an Observatory in space and a sophisticated ground system to enable efficient use of the Observatory. The key properties of the Observatory are:

- Telescope: $85 \mathrm{~cm}$ aperture Cassegrain operated at $5.5 \mathrm{~K}$.

- Cryostat: 360 liters of Superfluid Helium at 1.4K.

- Science Instruments:

1. Infrared Array Camera (IRAC), 3.6, 4.5, 5.8, and 8.0 micron bands each with $5 \times 5$ arc minute fields of view.

2. Infrared Spectrograph (IRS), with low resolution spectral capability between 5 and 40 microns and medium resolution spectral capability between 10 and 40 microns.

3. Multiband Imaging Photometer for STRTF (MIPS), with 24, 70, and 160 micron imaging capability, in addition to 50 to 100 micron spectral energy distribution capability.

- Pointing: Sub arc second pointing stability and offset accuracy.

- Orbit: Solar, earth trailing.

- Telecommunications: $X$ band up/down using NASA Deep Space Network.

The Observatory, shown below, will be the first large aperture infrared telescope that will be launched at earth ambient temperature, and cooled in space to cryogenic temperature. Cooling will be provided by a combination of radiation to space, and cryogen cooling provided by the vapor from boil off of superfluid helium. This technique will enable a large aperture cryogenic telescope to be put in space with a projected lifetime approaching five years using a Delta II launch vehicle.

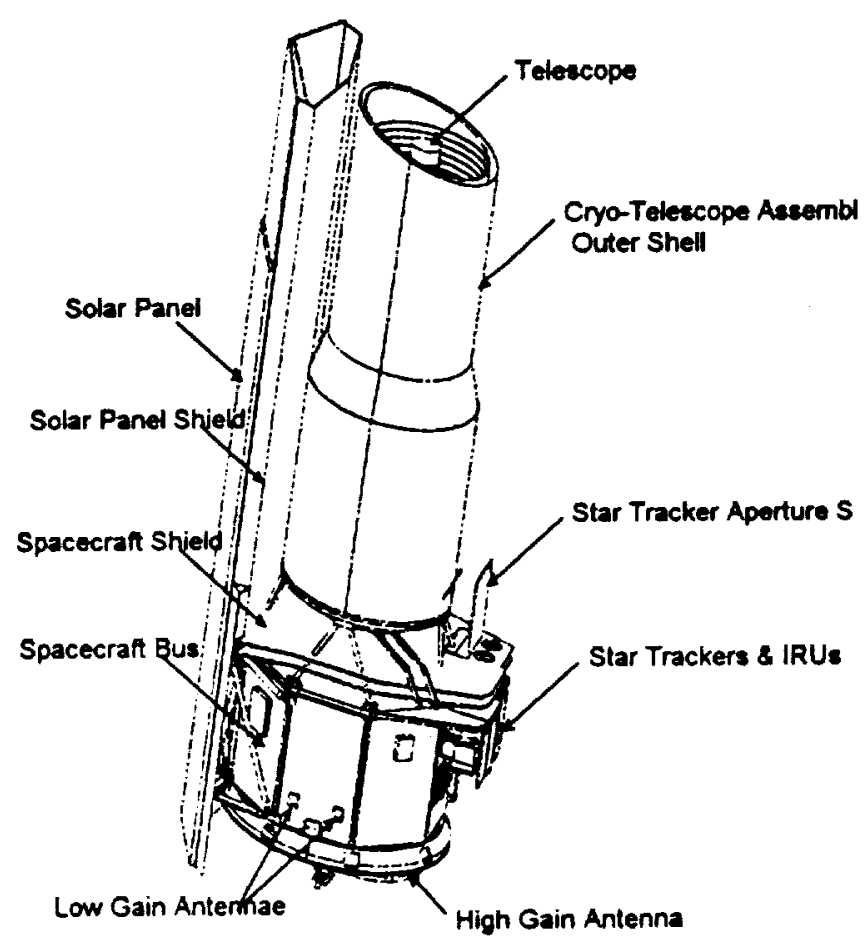


SIRTF will provide a capability to do background limited research in spectral regions not accessible from the earth's surface. Eleven (11) large arrays, sensitive from 3.5 to 180 microns will be available to collect both spatial and spectral information. The unprecedented sensitivity of a new generation of infrared array detectors will enable observations that have been previously unachievable either in space or from the ground. For example, SIRTF will measure sources which are 10,000 times fainter than the limits reached by the IRAS all sky infrared survey in 1983. For simplicity and reliability, SIRTF has only four (4) mechanisms that operate at cryogenic temperatures (not counting a deployable dust cover for the telescope and cryostat valves).

SIRTF will be placed in a solar orbit that will allow the Observatory to trail the Earth as it orbits the sun. The observatory will recede from the earth at about 0.13 AU/year. At this rate the Observatory is expected to be at about $0.64 \mathrm{AU}$ at the end of the 5 year planned mission. This orbit has many advantages. Among them are; maximum payload to orbit, no need for on-board propulsion system, no periodic solar eclipse by earth, no thermal radiation from earth, and excellent access to astronomical targets.

The Observatory will communicate with Earth periodically using the NASA Deep Space Network (DSN). Data are stored on board and downlinked every 12 hours. Operation of the Observatory will be conducted by on-board systems responding to preplanned sequences which are sent to the Observatory once a week. Data transmission will use $\mathrm{X}$ band systems, and will have a bit rate for the first 2.5 years of $2.2 \mathrm{Mbps}$. The spacecraft portion of the Observatory also provides solar collected power, pointing control, and command and data handling of the Observatory and its instruments.

The facility will be accessible by the entire international community through the SIRTF Science Center which will solicit investigations, coordinate all scientific observations, and will calibrate and distribute the resulting data. At least $75 \%$ of observing time will be awarded through peer reviewed proposals. Day by day control of the Observatory will be performed by a flight operations system that includes the NASA Deep Space Network, and multimission capabilities at JPL.

\section{The Cryogenic Telescope Assembly:}

Prior to SIRTF, infrared astronomy missions have been designed to cool both the instruments and telescope prior to launch, and then open a cover in space to commence observing. The penalty associated with this approach is that to maintain all of the needed equipment at cryogenic temperatures on the ground requires that they be placed in a vacuum container. As the size of the telescope aperture grows, so does the size (and mass) of the vacuum container. For SIRTF, an approach has been developed that allows the $85 \mathrm{~cm}$ Beryllium telescope to be at room temperature on the ground, and then cooled in space to S.SK by a combination of radiation, and superfluid helium boil off. As a result the vacuum container need only contain the cold portions of the instruments and the cryogen.

Radiation cooling is accomplished by shading the Observatory from the sun using the solar array. The warm spacecraft portion of the Observatory is isolated from the cold portion, known as the Cryogenic Telescope Assembly (CTA), using struts made of gamma-alumina. As a result of shielding and isolation, the telescope will cool to less than $40 \mathrm{~K}$ using radiation alone. Added cooling of the telescope is provided by thermally connecting the telescope to the lines carrying the vapor from the boil off of superfluid Helium. A resulting telescope temperature of 5.SK is obtained if the heat load on the helium is about 6 milliwatts. Since that is the design point for the cryogenic portions of each instrument, an efficient use of cryogen results. While the requirement for SIRTF is to sustain the cryogenic operations for 2.5 years, current estimates are that under nominal conditions a lifetime of 5 years can be expected with only 360 liters of Helium at launch.

The cryogenic portions of the instruments are contained within a Multiple Instrument Chamber (MIC) located in the cryostat. The cryostat is surrounded by shields designed to minimize the heat load on the cryogen. The shields are vapor cooled and/or radiate to space resulting in a cryostat with an outer vacuum shell operating at near $5 \mathrm{~K}$.

\section{The InfraRed Array Camera (IRAC):}

IRAC is a camera intended to provide high resolution and wide field imagery in four spectral bands. The camera is designed to have two optical paths, and each path contains a beamsplitter that sends short wavelength radiation to a $256 \times 256$ element Indium Antimonide (InSb) array, and the long wavelength radiation to a 256.256 element Arsenic doped Silicon (Si:As) array. One optical path provides images at 3.6 microns and 5.8 microns. The other path provides images at 4.5 microns and 8.0 microns. The only moving element of the camera is a shutter which will be used occasionally to close the aperture for calibration purposes. The IRAC uses programmed gate arrays to establish operating sequences. Commands from the spacecraft are used to establish bias voltages, and command readouts. The simplicity of the design is considered to be a strength for reliability and ease of operation.

The InfraRed Spectrograph (IRS): 
The IRS consists of four modules, each of which is a self contained spectrograph with a $128 \times 128$ element array in the focal plane. Two modules are low resolution spectrographs, one operating between 5 and 15 microns, and the other between 15 and 40 microns. The other two modules are medium resolution spectrographs operating between 10 and 20 microns, and between 20 and $40^{\circ}$ microns with orders folded to fully utilize the second dimension of each array. Both short wavelength spectrographs are read out using $128 \times 128$ element Si:As arrays. Similarly, at the longer wavelengths $128 \times 128$ element Antimony doped Silicon (Si:Sb) arrays are used.

In order to assure placement of the desired object on a selected spectrograph slit, a portion of one array will be used to form an image to peak-up the object. Peak-up srefers to the process of optimally placing the object on the selected slit. For SIRTF this will be done on-board using information about the location of the object derived from the peak-up array. This information will be processed by the spectrograph. The resulting data will be used to define a pointing offset, and the telescope will then be pointed such that the object falls on the selected spectrograph slit.

\section{The Multiband Imaging Photometer for SIRTF (MIPS):}

MIPS contains three arrays which are used primarily for imaging. It may also be used in a spectral energy distribution mode, and in a photometer mode to measure total power being observed. Unlike the other SIRTF instruments, MIPS is dependent upon a cryogenically cooled scanning mirror for proper operation. The shortest wavelength for MIPS is 24 microns, which is observed using a $128 \times 128$ element $\mathrm{Si}$ :As array. At 70 mictons a $32 \times 32$ element Gallium doped Germanium (Ge:Ga) array is employed. For the longest wavelengths being observed by SIRTF, out to about 180 microns, a $2 \times 20$ element Stressed Ge:Ga array is used. The MIPS pixels have been sized to permit critical sampling of the image from the 85 $\mathrm{cm}$ telescope. The scanning mirror has several facets, and allows for simultaneous observing with each of the three arrays. By repositioning the scanning mirror additional optics are introduced into the path that disperse the radiation through a low resolution grating resulting in a measurement of spectral energy distribution, the SED mode. Another position of the scanning mirror places MIPS in the total power mode.

The IRS and MIPS instruments use a common set of warm electronics which contains a RAD 6000 microprocessor that controls the operating instrument, and sends the resulting data to the spacecraft for packing. storage, and subsequent transmission to the earth.

\section{The Spacecraft:}

The primary functions of the spacecraft are to provide power, pointing, command and data handling, and telecommunications. Each function requires sophisticated on-board systems to assure high reliability and precision operation. Power is provided by Gallium Arsenide solar cells collecting solar energy and distributing it on a bus to internal subsystems, and on command to the designated instrument. A battery is included, primarily for launch operations, but also for additional protection in the event of an Observatory anomaly.

Pointing is provided using a combination of star tracker data, gyroscope data, and information from a Pointing Calibration Reference Sensor (PCRS) located in the cryogenic focal plane of the telescope. Reaction wheels are used to position the Observatory, with a nitrogen gas reservoir on board for momentum management. Both the star tracker and PCRS are sophisticated instruments which internally determine their pointing direction, and relay this information to the spacecraft computer which uses the data to determine how to point the telescope in the desired direction.

The spacecraft uses a RAD 6000 microprocessor based Command and Data Handling (C\&DH) subsystem that provides the computing power for the entire spacecraft. Instrument commands are issued by the $\mathrm{C} \& \mathrm{DH}$, and the resulting data is collected, compressed, packetized, and stored for transmission to earth in subsequent telecommunications sessions. Observing sequences are controlled by the C\&DH based on weekly updates from earth. The C\&DH also provides the processing capability needed to point the observatory, and maintain its position during observations and when communicating with earth.

Telecommunications are at $\mathrm{X}$ band frequencies using a 1.35 meter high gain antenna mounted at the base of the spacecraft. The antenna is fixed, and is pointed to the Earth by pointing the entire Observatory. When in communication with the Earth, data transmission occurs at rates up to $2.2 \mathrm{Mbps}$, and commands can be concurrently sent to the Observatory at a rate of $2 \mathrm{Kbps}$. A NASA developed Small Deep Space Transponder (SDST) prepares the packetized on board data for transmission, and sends the Reed-Solomon encoded convolutionally coded data to the X-band solid state power amplifiers. The SIRTF design provides for redundant transmitters at 15 watts that can be connected together for a non-redundant transmission capability of 29 watts. This feature will be used to extend the high data mate capabilities of SIRTF as the mission progresses and the distance from the earth to the Observatory increases.

Key SIRTF Tests prior to launch: 
The reliance on the SIRTF thermal design to achieve the required performance necessitates a robust test program. While the concept of radiative cooling is straight forward, its implementation is sophisticated. To assure that acceptable operating temperatures will be attained, and that the lifetime expected will be achieved, a thermal balance test is planned to demonstrate that the thermal design performs as specified. This test will require the use of a large Helium shroud to simulate the space environment for the entire CTA.

Optical performance is being provided using a technique not previously proven in space. Launching the SIRTF telescope at earth ambient temperature, and then cooling it in space to $5.5 \mathrm{~K}$ while requiring diffraction limited performance (at 6.5 microns) represents a eignificant optical challenge. To verify that the telescope will meet its requirements, an end-to-end optical test is planned following assembly of the CTA. The telescope will be cooled from earth ambient temperature to $5.5 \mathrm{~K}$ in a test chamber, and IRAC and IRS arrays will be illuminated to demonstrate that the telescope performs as required at cryogenic temperatures. Risk has been reduced by including a focus mechanism at the secondary mirror which will be used for ground testing, and can be used in space if needed.

In addition to these performance tests, a series of operational scenario tests will be conducted on the entire observatory prior to launch to assure that the ground and Observatory systems perform together as expected. The scenarios will verify command and data handling capabilities, the ability of ground systems to plan and load long sequences up to the Observatory, and the ability of the Observatory to execute them autonomously.

\section{The Ground System}

The SIRTF Ground System consists of two main elements; a Flight Operations System (FOS) which has real time responsibility for operation of the Observatory, and the Science Operations System (SOS) with responsibility for making the facility accessible to the user community.

The SOS issues periodic announcements to the user community, selects observations to be performed on the Observatory, and plans observing sequences that efficiently use the Observatory's resources. Once observations are made, and data are returned to earth, the SOS processes the data to remove instrumentation arifacts. The resulting data are delivered to the requesting user(s) and placed in a SIRTF archive for general use by the research community.

The FOS, working with the SOS, prepares weekly scquences for execution on the Observatory. Once agreed upon, the weekly sequences are up loaded to the Observatory concurrent with one of fourteen weekly downlink passes. Two times each day, data are acquired from the Observatory using the NASA DSN. The received data are decoded, unpacked, and the engineering data sent to monitoring teams for evaluation. The science data are sent to the SOS for processing and distribution.

\section{The SIRTF Team:}

SIRTF is being developed using a team approach. The Jet Propulsion Laboratory has overall project responsibility and is the team leader, in addition JPL is responsible for the Flight Operation System development. The Smithsonian Astrophysical Observatory is providing the IRAC instrument with hardware being provided by the NASA Goddard Space Flight Center. Cornell University is providing the IRS instrument with hardware being provided by the Ball Aerospace and Technology Corporation. The University of Arizona is providing the MIPS instrument with hardware being provided by the Ball Aerospace and Technology Corporation. The Ball Aerospace and Technology Corporation is also providing the Cryogenic Telescope Assembly. Lockheed Martin Missiles and Space is providing the Spacecraft, system engineering support, and Observatory level integration, test and launch coordination. The Science Operation System development is the responsibility of the Califormia Institute of Technology.

Additional information on SIRTF can be obtained by accessing SIRTF information on the World Wide Web at http://SIRTF.caltech.edu.

The research described in this paper was carried out by the Jet Propulsion Laboratory, California Institute of Technology, under a contract with the National Aeronautics and Space Administration. 